\title{
Membrane-bound GFP-labelled vectors for Targeted DamID allow simultaneous profiling of expression domains and DNA binding
}

\author{
Caroline Delandre $^{1}$, John PD McMullen ${ }^{1}$ and Owen J Marshall ${ }^{1 *}$
}

April 17, 2020

\begin{abstract}
Targeted DamID (TaDa) allows highly efficient celltype-specific profiling of protein-DNA interactions. Cell-type-specificity, however, is governed by the GAL4/UAS system, which can exhibit differences in expression patterns depending upon the genomic insertion site and the UAS promoter strength. The $\mathrm{TaDa}$ system uses a bicistronic transcript to reduce the translation rates of Dam-fusion proteins, presenting the possibility of using the primary ORF within in the transcript to label expression domains and precisely identified the profiled cell populations in experimental samples. Here, we describe two TaDa vectors, $\mathrm{pTaDaG}$ and $\mathrm{pTaDaG} 2$, that use myristoylated GFP as the primary ORF. Differing lengths of the myristoylation sequence between the plasmids allows additional translational control. Fly lines created with this system allow easy visualisation of expression domains under both fluorescent dissecting and confocal microscopes without the use of antibody staining, whilst faithfully profiling protein-DNA interactions via Targeted DamID.
\end{abstract}

\section{Introduction}

Targeted DamID (TaDa) is a recently developed technique that generates in vivo cell-type-specific binding profiles of DNA-binding, chromatinmodifying, or DNA-associated proteins in Drosophila melanogaster $[1,2]$. The technique is highly reproducible and extremely sensitive, generating binding profiles from as few as 10,000 cells in living

\footnotetext{
*Corresponding author: owen.marshall@utas.edu.au ${ }^{1}$ Menzies Institute for Medical Research, University of Tasmania, 17 Liverpool St, Hobart TAS 7000, Australia.
}

organisms [2]. The technique is a variant of DamID, in which a protein of interest is fused to DNA Adenine Methylase (Dam) from Escherichia coli, leading to the enriched methylation of GATC sites in close proximity to where the protein of interest binds.

In TaDa, cell-type-specificity is accomplished via the GAL4/UAS system [3]. High levels of cellular Dam, however, are toxic; and a signature feature of TaDa is a significant reduction in the translation levels of Damfusion proteins from highly-expressed GAL4-driven transcripts [1]. In TaDa, this lowering of translation levels is accomplished via a bicistronic transcript, where an upstream ORF is separated from the Dam-fusion ORF by two stop codons and a frameshift. The Damfusion ORF is thus only translated via spontaneous ribosome re-initiation, in which the rates of translation of a secondary ORF are inversely proportional to the length of the primary ORF [4]. The original TaDa system used full-length mCherry as the primary ORF. In theory, this presents the possibility of visualising the profiled cell population via microscopy of dissected experimental tissue; however, the low brightness of mCherry and the lack of localisation to a particular cellular compartment makes visualisation of expression domains in $\mathrm{TaDa}$ experimental samples challenging in practice.

Although broadly defined expression patterns for GAL4 drivers are known, the precise expression pattern and the amount of driver background from other tissues depends upon the targeted insertion site and UAS promoter strength [5]. Given the sensitivity of the TaDa technique and that only a small subset of the cells within an isolated tissue are typically profiled within a TaDa experiment, knowing the exact cells profiled with a GAL4 driver in the TaDa system is critically important to data interpretation. 
Here, we describe two new TaDa vectors that use membrane-targeted myristoylated-GFP as a primary ORF. The TaDaG vector uses an 85 amino acid (aa) myristoylation sequence; TaDaG2 uses a 14 aa minimal myristoylation sequence. The differences in the primary ORF allow differing secondary ORF translation levels, but otherwise behave identically. Importantly, these primary ORFs allow easy fluorescent identification of profiled cell populations within the experimental sample. The vectors also incorporate a StuI restriction site upstream of Dam to easily facilitate the creation of C-terminal Dam-fusion proteins.

\section{Methods}

\section{Expression constructs}

The $p T a D a G$ vector was constructed by cutting pUASTattB [6] with EcoRI and XbaI, and inserting a 1969 bp custom gBlock (IDT) containing EcoRImyrGFP-StuI-Dam-MCS-XbaI via NEB HiFi assembly (NEB). The myrGFP sequence represents the first 85 aa of D. melanogaster Scr64B fused to a D. melanogastercodon-optimised GFP ${ }^{\mathrm{F} 64 \mathrm{~L}, \mathrm{~S} 65 \mathrm{~T}, \mathrm{H} 231 \mathrm{~L}}$ [5]. The $p T a D a G 2$ vector was created by cutting the $p T a D a G$ with EcoRI and NdeI, and inserting a 350bp gBlock (IDT) containing the minimal 14aa myristoylation sequence MGSSKSKPKDPSQR from $\mathrm{p} 60^{\mathrm{src}}$ [7] and the 5' portion of GFP, again via NEB HiFi assembly. pTaDaG$P c$ was generated by cutting $p T a D a G$ with $\mathrm{BglII} / \mathrm{XhoI}$ and inserting a 1233bp gBlock (IDT) containing the $P c-R A$ ORF. All plasmids were sequence-verified via Sanger sequencing (ABI). Plasmid maps were generated using SnapGene software (Insightful Science).

\section{Fly lines}

GAL4 driver lines used were worniu-GAL4 [8] for neural stem cells and R13F02-GAL4 [9] for Mushroom body neurons. Lines were crossed to a tub-GAL80ts stock to generate a worniu-GAL4;tub-GAL80ts line.

TaDaG-Dam, TaDaG2-Dam and TaDaG-Pc fly lines were generated by BestGene, Inc (CA), through phiC31-integrase-mediated insertion of the appropriate expression vectors into attP2 on chromosome $3 \mathrm{~L}$.

\section{Confocal microscopy}

Larval brains (3rd instar, 96hrs ALH) were dissected in PBS and fixed in PBS $+0.3 \%$ TritonX-100 (PBST) with $4 \%(\mathrm{v} / \mathrm{v})$ paraformaldehyde (ProSciTech) for 20 mins, $4^{\circ} \mathrm{C}$, before three $10 \mathrm{~min}$ washes in PBST. Brains were mounted in Vectorshield + DAPI, and imaged under an Olympus FV3000 confocal microscope at 20x

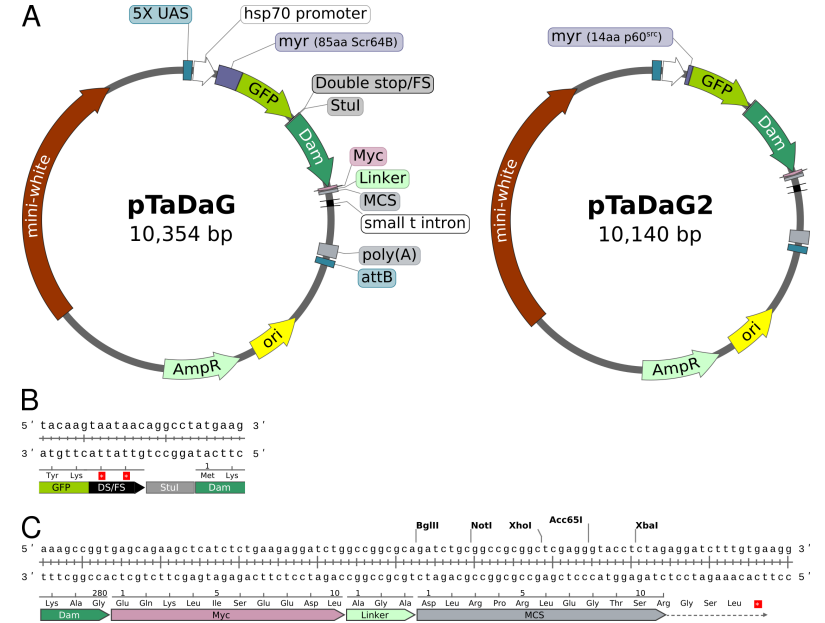

Figure 1: The pTaDaG and pTaDaG2 plasmids. (A) Plasmid maps illustrating the shared features between both plasmids on $\mathrm{pTaDaG}$ and the differing myristoylation domains. (B) Sequence of the linker region between the primary GFP and secondary DAM ORFs. (C) Sequence of the Myc-tag-linker-MCS region.

magnification.

\section{Targeted DamID}

TaDaG-Dam or TaDaG-Pc males were crossed to worniu-GAL4;tub-GAL80ts virgin females in cages. Embryos were collected on apple juice agar plates with yeast over a 4-hour collection window at $25^{\circ} \mathrm{C}$ and grown at $18^{\circ} \mathrm{C}$ for two days. Newly hatched larvae were transferred to food plates for a further five days at $18^{\circ} \mathrm{C}$, before shifting to $29^{\circ} \mathrm{C}$ for 24 hours. Larval brains were dissected in PBS, and processed for DamIDseq as previously described $[2,10]$ with the following modifications. Briefly, DNA was extracted using a Quick-DNA Miniprep plus kit (Zymo), digested with DpnI (NEB) overnight and cleaned-up with a PCR purification kit (Machery-Nagel), DamID adaptors were ligated, digested with DpnII (NEB) for 2 hours, and amplified via PCR using MyTaq DNA polymerase (Bioline). Following amplification, $2 \mu \mathrm{g}$ DNA was sonicated in a Bioruptor Plus (Diagenode), DamID adaptors removed by AlwI digestion, and 500ng of the resulting fragments end-repaired with a mix of enzymes (T4 DNA ligase (NEB) + Klenow Fragment (NEB) + T4 polynucleotide kinase (NEB)), A-tailed with Klenow 3' to 5' exo- (NEB), ligated to Illumina Truseq LT adaptors using Quick Ligase enzyme (NEB) and amplified via 

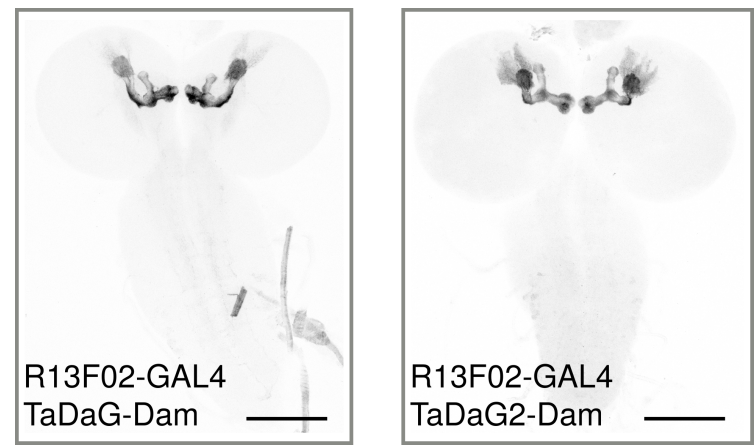

Figure 2: myrGFP labelling of 3rd instar larval mushroom body neurons via the $\mathrm{TaDaG}$ systems. TaDaG-Dam or TaDaG2-Dam flies were crossed to the mushroom-body-specific R13F02-GALA driver line. Maximum image projections from confocal stacks are shown. Scale bar: $100 \mu \mathrm{m}$.

PCR with NEBNext Hi-fidelity enzyme (NEB).

The resulting next-generation sequencing libraries were sequenced on a HiSeq2500 and reads were processed with damidseq_pipeline [11].

\section{Bioinformatic analysis}

Binding profiles were visualised using pyGenomeTracks [12]. Heatmaps were generated via the ComplexHeatmap R package [13]. All other plots were generated using $\mathrm{R}$ [14].

\section{Results and Discussion}

\section{Design of the TaDaG and TaDaG2 vectors}

The $p T a D a G$ and $p T a D a G 2$ plasmids were generated from $p U A S T a t t B$ using synthetic DNA. In designing the myrGFP insert, we combined Drosophila codonoptimised GFP ${ }^{\mathrm{F} 64 \mathrm{~L}, \mathrm{~S} 65 \mathrm{~T}, \mathrm{H} 231 \mathrm{~L}}$ [5] with either the 85aa myristoylation sequence from Scr64B [5] (pTaDaG) or a 14 aa minimal $\mathrm{p} 60^{\text {src }}$ myristoylation sequence [7] (pTaDaG2) (Fig. 1A). Following the primary ORF, we incorporated a double stop codon / single base frameshift linker, as per the original $\mathrm{TaDa}$ vector (pUAST-attB-mCherry-NDam) [1], together with a StuI restriction enzyme site upstream and in-frame with Dam to allow easy generation of C-terminal Dam fusion proteins (Fig. 1B). Spacing between ORFs is reported to have little effect on translation rates of the secondary ORF [4], allowing the incorporation of the StuI site with no translational penalty. The MCS region is separated from Dam using the same Myc-tag+linker sequence as the original vector, allowing cloning compatibility
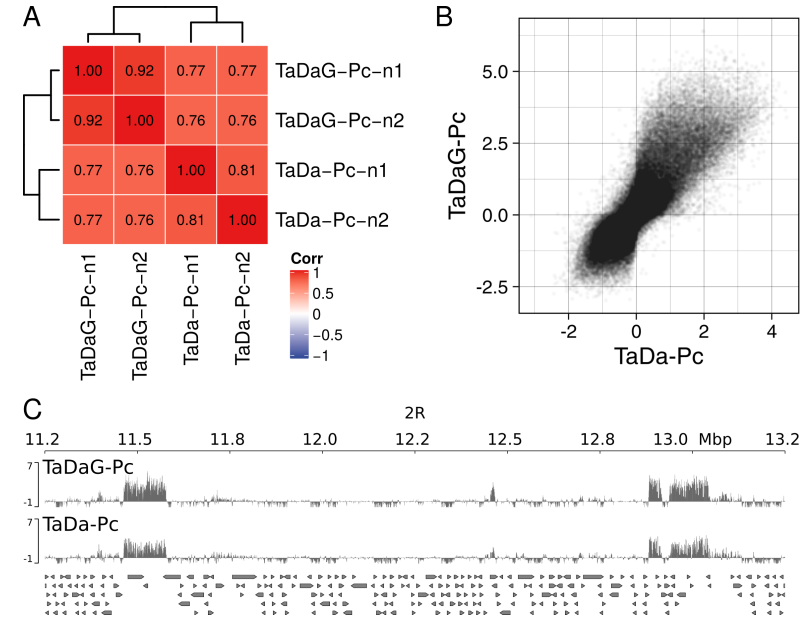

Figure 3: DamID profiling of Polycomb binding in 3rd instar larval NSCs using the TaDaG system. (A) Pearson's correlation of TaDaG-Pc biological replicates vs previously published TaDa-Pc replicates [10]. (B) Correlation plot of the average Polycomb binding enrichment over all genomic GATC fragments for TaDaG-Pc and TaDa-Pc profiles. (C) TaDaG-Pc and TaDa-Pc binding over two canonical Polycomb foci on chromosome 2R. Scores for (B) and (C) represent normalised $\log _{2}$ (Dam-fusion/Dam) binding enrichment.

between vectors (Fig. 1C).

TaDaG and TaDaG2 label GALA-driven cell populations in vivo

In order to test the labelling capacity of the myrGFP primary ORFs in the $p T a D a G$ and $p T a D a G 2$ constructs, we crossed TaDaG-Dam and TaDaG2-Dam flies to the mushroom body neuron driver R13F02-GALA (Fig. 2). Both constructs exhibited clear and specific membranebound GFP labelling of mushroom body neurons that was detectable through native fluorescence (without antibody labelling) and was also visible under a fluorescent dissecting microscope (not shown).

The two myrGFP-labelled variants of the TaDa system allow simple verification of the expression pattern of Dam-fusion proteins under experimental conditions. The system also allows experimental crosses to be checked for correct GFP labelling during tissue collection. 
The TaDaG system faithfully profiles Polycomb binding domains in neural stem cells

To determine if the $\mathrm{TaDaG}$ system could generate cell-type-specific Targeted DamID profiles, we profiled Polycomb binding in neural stem cells (NSCs) using the NSC-specific driver worniu-GAL4, inducing expression for 24hours in 30 brains ( 9000 total profiled neural stem cells) in early (96hrs ALH) 3rd instar larvae (Fig. 3A). Two independent biological replicates had a very high correlation (Pearson's correlation between $\mathrm{TaDaG}$ replicates: 0.92 ), indicating excellent reproducibility even from very small sample sizes, and no loss of sensitivity when compared to the original TaDa system.

We compared the binding profiles to our previously published Polycomb binding data in NSCs obtained through the original TaDa system [2] (generated using a 16 hour induction timeframe rather than 24 hours in the current study). We observed a high correlation (minimum Pearson's correlation: 0.77) between the $\mathrm{TaDa}$ and TaDaG-generated profiles (Fig. 3A,B) and clear concordant binding over canonical Polycomb foci (Fig. 3C), indicating that the TaDaG system functions indistinguishably from the original TaDa constructs.

Given that GAL4-driver expression patterns are dependent upon both the insertion site and the UAS promoter sequence [5], the ability to verify driver expression in situ is vital for determining the profiled cell population in Targeted DamID and interpreting subsequent binding data. We anticipate that these vectors will prove highly useful to the community.

\section{Acknowledgments}

We thank G. Jefferies for technical assistance. This work was supported by an NHMRC grant APP1128784 to OJM and an Ian Potter Foundation equipment grant (20190091) to OJM.

\section{References}

1. Southall TD, Gold KS, Egger B, et al. Dev. Cell, 26(1):101-12, 2013.

2. Marshall OJ, Southall TD, Cheetham SW, et al. Nat. Protoc., 11(9):1586-1598, 2016.

3. Brand $\mathrm{AH}$ and Perrimon N. Development, 118(2):401-15, 1993.

4. Luukkonen BG, Tan W, and Schwartz S. J. Virol., 69(7):4086-4094, 1995.
5. Pfeiffer BD, Ngo TTB, Hibbard KL, et al. Genetics, 186(2):735-55, 2010.

6. Bischof J, Maeda RK, Hediger M, et al. Proc. Natl. Acad. Sci. U. S. A., 104(9):3312-3317, 2007.

7. Pellman D, Garber EA, Cross FR, et al. Nature, 314(6009):374-377, 1985.

8. Albertson R, Chabu C, Sheehan A, et al. J. Cell Sci., 117(Pt 25):6061-70, 2004.

9. Jenett A, Rubin GM, Ngo TTB, et al. Cell Rep., 2(4):991-1001, 2012.

10. Marshall OJ and Brand AH. Nat. Commun., 8(1):2271, 2017.

11. Marshall OJ and Brand AH. Bioinformatics, 31(20):3371-3, 2015.

12. Ramírez F, Bhardwaj V, Arrigoni L, et al. Nat. Commun., 9(1), 2018.

13. Gu Z, Eils R, and Schlesner M. Bioinformatics, 32(18):2847-2849, 2016.

14. R Development Core Team. R: A Language and Environment for Statistical Computing, 2011. 